Research

\title{
DCE-MRI radiomics nomogram can predict response to neoadjuvant chemotherapy in esophageal cancer
}

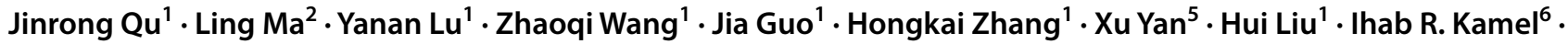 \\ Jianjun Qin ${ }^{3,4} \cdot$ Hailiang $\mathrm{Li}^{1}$
}

Received: 28 September 2021 / Accepted: 31 December 2021

Published online: 08 January 2022

(c) The Author(s) 2022 OPEN

\begin{abstract}
Objectives To assess volumetric DCE-MRI radiomics nomogram in predicting response to neoadjuvant chemotherapy (nCT) in EC patients.

Methods This retrospective analysis of a prospective study enrolled EC patients with stage $\mathrm{CT} 1 \mathrm{~N}+\mathrm{M} 0$ or cT2-4aN0-3M0 who received DCE-MRI within 7 days before chemotherapy, followed by surgery. Response assessment was graded from 1 to 5 according to the tumor regression grade (TRG). Patients were stratified into responders (TRG1 +2$)$ and nonresponders (TRG3 $+4+5) .72$ radiomics features and vascular permeability parameters were extracted from DCE-MRI. The discriminating performance was assessed with ROC. Decision curve analysis (DCA) was used for comparing three different models.

Results This cohort included 82 patients, and 72 tumor radiomics features and vascular permeability parameters acquired from DCE-MRI. mRMR and LASSO were performed to choose the optimized subset of radiomics features, and 3 features were selected to create the radiomics signature that were significantly associated with response $(P<0.001)$. AUC of combining radiomics signature and DCE-MRI performance in the training $(n=41)$ and validation $(n=41)$ cohort was 0.84 (95\% Cl 0.57-1) and $0.86(95 \% \mathrm{Cl} 0.74-0.97)$, respectively. This combined model showed the best discrimination between responders and non-responders, and showed the highest positive and positive predictive value in both training set and test set.

Conclusions The radiomics features are useful for $\mathrm{nCT}$ response prediction in EC patients.
\end{abstract}

Keywords Esophageal cancer · Magnetic Resonance Imaging · Adjuvant chemotherapy · Nomograms · Precision medicine
Abbreviations
TRG Tumor regression grade
DCA Decision curve analysis
EC Esophageal cancer

Jinrong Qu and Ling Ma contributed equally to this work

\begin{abstract}
$\triangle$ Jianjun Qin, 123114172@qq.com| ${ }^{1}$ Department of Radiology, Affiliated Cancer Hospital of Zhengzhou University and Henan Cancer Hospital, Zhengzhou 450008, Henan, China. ${ }^{2}$ Advanced Application Team, GE Healthcare, Shanghai 201203, China. ${ }^{3}$ Department of Thoracic Surgery, Affiliated Cancer Hospital of Zhengzhou University, Zhengzhou 450008, Henan, China. ${ }^{4}$ Present Address: Department of Thoracic Surgery, National Cancer Center/Cancer Hospital, Chinese Academy of Medical Sciences and Peking Union Medical College, Beijing 100021, China. ${ }^{5}$ NEA MR Collaboration, Siemens Ltd., China, Shanghai 201318, China. ${ }^{6}$ Department of Radiology, Johns Hopkins University School of Medicine, Baltimore, MD 21205-2196, USA.
\end{abstract}




$\begin{array}{ll}\text { PCR } & \text { Pathologic complete response } \\ \text { DCE } & \text { Dynamic contrast enhanced } \\ \text { EGD } & \text { Esophagogastroduodenoscopy } \\ \text { AIF } & \text { Arterial input function } \\ \text { ROIs } & \text { 3D regions of interests } \\ \text { ICC } & \text { Inter-class correlation coefficient } \\ \text { VIF } & \text { Variance inflation factor } \\ \text { AUC } & \text { Area under curve } \\ \text { RLM } & \text { Run-length matrix } \\ \text { nCT } & \text { Neoadjuvant chemotherapy }\end{array}$

\section{Introduction}

Esophageal cancer (EC) is the sixth leading cause of cancer mortality globally [1]. Neoadjuvant therapy combined with surgery has become the standard treatment for local advanced EC [2, 3], and which includes neoadjuvant chemoradiotherapy $(n C R T)$ and neoadjuvant chemotherapy $(n C T)[4,5]$. Although it has been reported that $n C R T$ could achieve more pathologic complete response ( $\mathrm{pCR}$ ) than $\mathrm{nCT}$, an updated meta-analysis showed no clear advantage of $\mathrm{nCRT}$ over $\mathrm{nCT}$ [6]. In western countries, ECs are mainly adenocarcinoma, and nCRT is the treatment of choice [7]. However, in China and Japan, almost all the ECs are squamous cell carcinoma, and $\mathrm{nCT}$ is the treatment of choice particularly for stages II and III [5]. Although $\mathrm{nCT}$ could improve overall survival, surgery is still important especially for non-responsive patients [8]. However, pretreatment prediction of response to $\mathrm{nCT}$ in EC remains challenging.

Radiomics could provide more information than conventional images, and pretreatment 18F-FDG PET and MRI radiomics have been used for predicting outcome of patients with locally advanced cervical cancer treated with chemoradiotherapy [9], as well as in rectal cancer [10]. Meanwhile, MRI radiomics showed individualized estimation of lymph node metastasis in EC patients [11]. Dynamic contrast enhanced (DCE)-MRI represents tumor perfusion by contrast media, and it has an encouraging role in predicting tumor response to $n C R T$ and patient survival $[4,12]$. Recent improvements in DCE-MRI allow for high quality imaging of the chest during free-breathing. However, to date there is no report about volumetric DCE-MRI radiomics nomogram that could predict response of EC patients to $\mathrm{nCT}$. Therefore, our goal was to develop and validate such a volumetric DCE-MRI signature of primary tumor and determine if this signature could predict tumor histopathologic response in patients with EC treated with nCT.

\section{Methods}

\subsection{Patients}

Pretreatment staging was evaluated with measures including physical examination, standard laboratory tests, pulmonary function tests, esophagogastroduodenoscopy (EGD) with endoscopic ultrasound and biopsy, chest/abdominal CT with contrast, and PET if available. A multidisciplinary team was employed for patients' evaluation before treatment, as stated by institutional practice guidelines between September 2016 and March 2018. Patients had histologically proven and resectable EC with stage $\mathrm{CT} 1 \mathrm{~N}+\mathrm{MO}$ or $\mathrm{CT} 2-4 \mathrm{aNO}-3 \mathrm{MO}$, according to the 7th TNM staging system of the American Joint Committee on Cancer [13]. The consecutive patients were retrospectively evaluated, and this analysis of a prospective study (ChiCTR-DOD-14005308) was approved by the institutional review board and informed consent was performed by all patients.

\subsection{MRI technique}

All patients received DCE-MR before chemotherapy, which was performed within 1 week before the beginning of chemotherapy, and the time interval between initial staging and pretreatment MRI was 1-6 days. 


\subsection{Positioning coil selection and examination}

MRI examination in a 3 T MR scanner (MAGNETOM Skyra, Siemens Healthcare, Erlangen, Germany) with DCE radial VIBE with $3 \mathrm{~mm}$ slice thickness. A prototype dynamic radial VIBE sequence with a total of 68 periods and 4896 images were collected, as every period included 72 images, was performed for the whole chest during free breathing with the total scanning time of $309 \mathrm{~s}$. At $20 \mathrm{~s}$ after the beginning of scanning, 10-15 mL Gadopentetate Dimeglumine Injection (0.2 ml/ $\mathrm{kg}$ of body weight, Omniscan, GE Healthcare) was injected at a rate of $2.5 \mathrm{~mL} / \mathrm{s}$ by a MR-compatible automated doubletube high-pressure injector (Spectris Solaris EP, Medrad, Indianola, PA), and equal volume of normal saline solution was used to flush the tube.

\subsection{Chemotherapy protocol}

All patients received 2 cycles of standard neoadjuvant paclitaxel and nedaplatin protocol followed by surgical resection, as published in a prior study [14]. The median time from the completion of chemotherapy to surgery was 23 days (range 21-30 days).

\subsection{Pathology}

Tumor regression grade (TRG) as described by the Mandard classification [15] was used to classify chemotherapy response assessment into 5 stages, including: TRG1 (no residual cancer cells), TRG2 (rare residual cancer cells), and TRG3 (fibrosis outgrowing residual cancer), TRG4 (residual cancer outgrowing fibrosis), and TRG5 (absence of regressive changes). A total of 82 patients had TRG available for this study and they were stratified into a responsive group (TRG1 +2$)$ and a non-responsive group (TRG3 $+4+5)$.

\subsection{Radiomics workflow}

Radiomics workflow of this study is presented in Fig. 1, including the following procedures: (1) DCE-MRI scanning, (2) chemotherapy, (3) DCE-MRI scanning, (4) vascular permeability parameters extraction, (5) radiomics feature extraction, (6) Radscore building, (7) building model and validating model.

\subsection{Tumor segmentation}

MR signal is usually relative, with large differences between scanners and vendors. By normalizing the image before feature calculation, this confounding effect may be reduced. The equation of image standardization as follow:

$$
\begin{gathered}
\text { Image standardization }=\frac{x-\mu}{\text { adjusted stddec }} \\
\text { adjusted stddev }=\max \left(\sigma, \frac{1.0}{\sqrt{ } N}\right)
\end{gathered}
$$

$\mu$ is the average level of signal for images. $X$ is the matrix of images. All images have been resampled to $1 \times 1 \times 1 \mathrm{~mm}^{3}$. DCE-MRI features analysis was performed on motion corrected data with an in-house developed software named Omnikinetics (GE Healthcare, China). Arterial input function (AIF) was obtained by placing a regions of interest (ROI) on the thoracic aorta in the peak arterial enhancement phase. An Extended Tofts Linear model was used to generate the permeability parameters. 3D tumors of pre-nCT were segmented by two radiologists (with 15 and 12 years of experience in MR imaging) by consensus (Appendix Fig. 6), who were blinded to the pathology results. The reviewers carefully segmented the entire tumor by manually contouring the whole tumor on each tumor slice. All large vessels and/or necrotic area(s) were avoided. The resulting 3D ROIs were used for subsequent extraction of radiomic features, and vascular permeability parameters were recorded. 


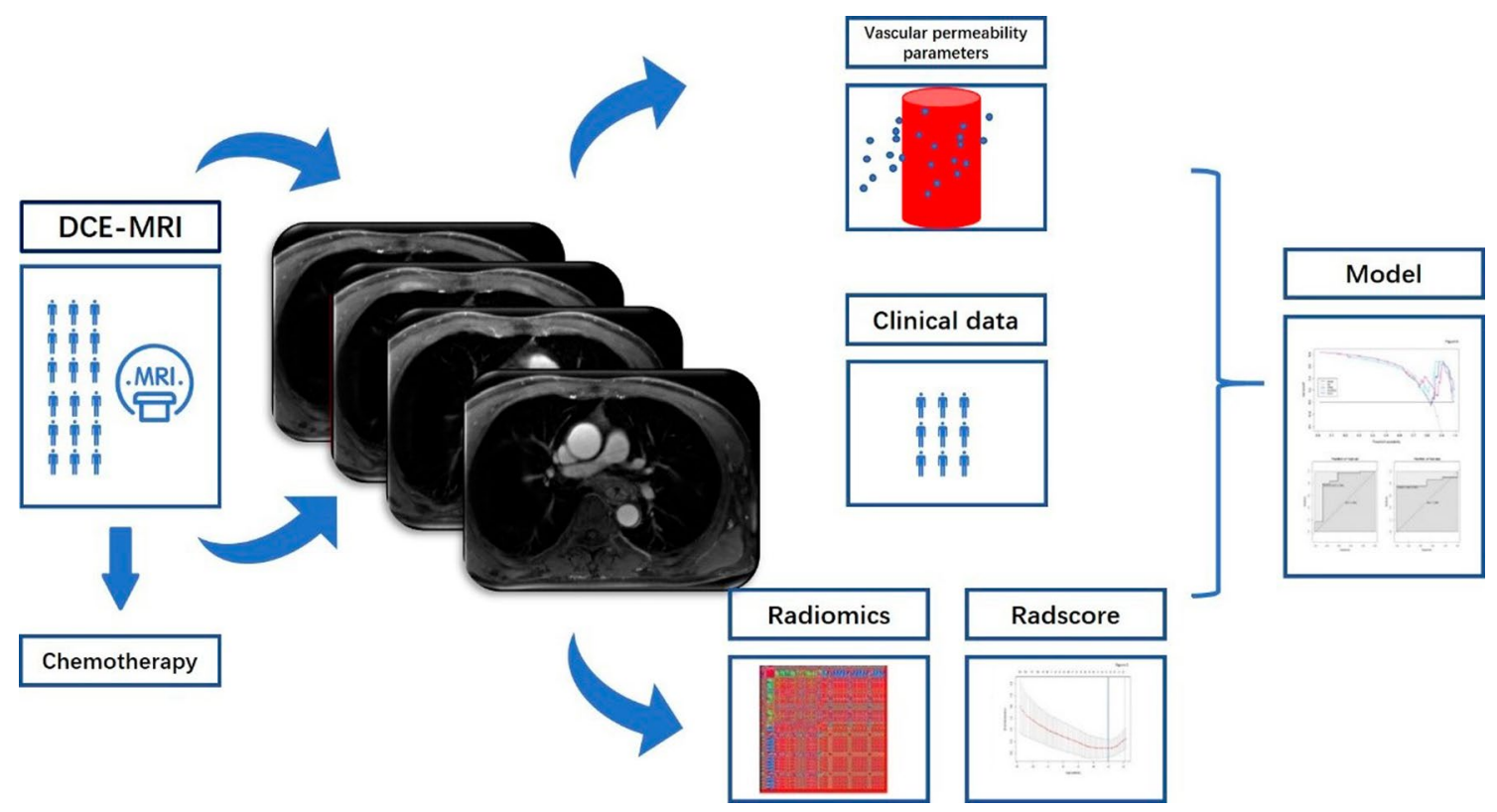

Fig. 1 Radiomics workflow

\subsection{Radiomics feature extraction}

Radiomics features were computed based on the 3D ROls segmentation results using a locally developed scientific software Omni-kinetics (GE Healthcare, Shanghai, China) that is not commercially available. Features were categorized into five primary subgroups: (1) 14 first-order, (2) 13 histogram, (3) 13 GLCM, (4) 16 RLM, and (5) 16 pharmacokinetic parameters. Intra-reader agreement of each radiomics feature was assessed by Inter-class correlation coefficient (ICC), and ICC with greater than 0.70 was considered a good agreement.

\subsection{Statistics analysis}

This group of research uses R (version 3.8.1) for processing and analysis, and uses "xml2" (read data), "tidyverse" (data visualization, data cleaning), "caret" (data preprocessing, feature selection), and "pROC" (model evaluation)), "glmnet" (LASSO, Logistic model), "DMwR" (uneven sample classification), "rmda" (clinical decision curve), "ggpubr" (data grouping), "ModelGood" (model evaluation), "rms" (Nomogram drawing), "mRMRe" (mRMR), "DescTools" (descriptive statistical analysis), "Publish" (logistic regression result) data package. Kologoroy Smirnov is used to test whether the measurement data conform to the normal distribution. The measurement data conforming to the normal distribution are represented by the mean \pm standard deviation, and the measurement data not conforming to the normal distribution are represented by the median. Enumeration data were compared by $\mathrm{X} 2$ test, and measurement data were compared by independent sample $t$ test or Mann-Whitney $U$ test. $P<0.05$, the difference is statistically significant. The effectiveness of the scoring system was evaluated based on the area under the ROC curve AUC.

\subsection{Dataset split and demographic comparison}

According to the ratio of 5:5, one half of this cohort (41 patients including 35 non-responders and 6 responders) was set as the training cohort, and the other half ( 41 patients including 35 non-responders and 6 responders) was set as the test cohort. We used independent samples t-test and Mann-Whitney $U$ test to evaluate the differences in age between the training set and test set, while chi-square tests were used to compare the differences in TRG, clinical characteristics and combined clinical characteristics and TRG (gender, clinical T-stage, N-stage, type, tumor location, and TRG). 


\subsection{Features redundancy and radiomic signature building}

Due to the high dimensionality of these imaging texture features, we only used stable features with ICCs value $\geq 0.70$ since these variables had good reproducibility.

The selected texture features were identified by one-way analysis of variance, Mann-Whitney $U$ test for classifying responders and non-responders in the training set. We used two feature selection method, mRMR and LASSO to select the feature. At first, mRMR was performed to eliminate the redundant and irrelevant features, and 30 features were retained. Then LASSO was conducted to choose the optimized subset of features to construct the final model. In order to assess the predicting performance of radiomics for each patient, a radiomics score (referred to as Rad-score) was computed for each patient, and the weight of each feature is determined by LASSO logistic with tenfold cross-validation which were obtained from the training set. The difference of Rad-score between the training and test set was analyzed using Mann-Whitney $\mathrm{U}$ (non-parametric, unpaired) test. We also performed univariable association analysis between the Radscore and response in both training and test sets.

\subsection{Radiomics nomogram development}

Multimodal logistic regression analysis started with a responsive group and a non-responsive group, clinical characteristics with the following candidate variables: gender, age, clinical T-stage, clinical N-stage, type and tumor location, and combined clinical characteristics and TRG. In order to provide a clinically useful tool which is able to predict the pathologic response to $\mathrm{nCT}$ probability of each patient, three nomograms of radiomics, DCE-MRI, and combined radiomics and DCEMRI were generated based on multivariate analysis in the training set. All the features of DCE-MRI would be included in the multimodal logistic regression model based on the variance inflation factor (VIF) that the threshold value of VIF is 10.

\subsection{Evaluation of prediction performance of the nomogram}

The performance of the resulting radiomics nomogram were first evaluated using area under curve (AUC) in the training set and then validated in the test set. The Mann-Whitney $U$ test was adopted for testing the potential correlation of the radiomics signature and response in the training cohort, and decision curve analysis (DCA) was used for comparing three models that were built with Radscore, DCE-MRI and the combination of Radscore and DCE-MRI. The positive predictive value (PPV) and negative predictive value (NPV) of different models were calculated.

\section{Results}

\subsection{Patient characteristics}

Patient characteristics in the training set and test set are shown in Table 1, and responders both in the training set and test set was $14.6 \%(6 / 41)$. According to independent samples $t$-test, there were no significant differences in demographic characteristics between the training set and test set.

\subsection{Selection of candidate radiomics features and building a radiomics signature}

After feature dimension reduction, 3 features were used to construct Rad-score which is a radiomics signature [16]. These 3 features were MinIntensity, LowGreyLevelRunEmphasis, ClusterShade, and were selected for classifying responders and non-responders in the training set (Fig. 2). The Rad-score was calculated according to the following formula: (Fig. 3)

$$
\text { Rad }- \text { score }=0.498 * \text { MinIntensity }+-0.11 * \text { ClusterShade }+-0.14 * \text { LowGreyLevelRunEmphasis }+1.887
$$

The radiomics signatures showed the significant difference between responsive group and non-responsive group in the Training set $(p=0.01$, Fig. $3 A$ ) and Testing set $(0.023$, Fig. $3 B$ ) with the AUC is 0.82 and 0.79 in Training set (Fig. $3 C$ ) and Testing set (Fig. 3D). 
Table 1 Patient and treatment-related characteristics with pre-nCT EC in the training set and test set $(n=82)$

\begin{tabular}{|c|c|c|c|c|c|}
\hline & \multicolumn{2}{|l|}{ Training set } & \multicolumn{2}{|l|}{ Test set } & \multirow[t]{2}{*}{$P$} \\
\hline & responders & non-responders & responders & non-responders & \\
\hline Gender & & & & & 0.563 \\
\hline Male & 3 & 32 & 5 & 18 & \\
\hline Female & 3 & 3 & 1 & 17 & \\
\hline Age, years & $56.8 \pm 9.4$ & $59.4 \pm 7.9$ & $62.0 \pm 7.6$ & $59.8 \pm 7.9$ & 0.545 \\
\hline Clinical T-stage & & & & & 0.198 \\
\hline T1 & 0 & 0 & 1 & 0 & \\
\hline $\mathrm{T} 2$ & 1 & 7 & 3 & 8 & \\
\hline T3 & 5 & 23 & 2 & 24 & \\
\hline T4 & 0 & 5 & 0 & 3 & \\
\hline Clinical N-stage & & & & & 0.429 \\
\hline No & 5 & 18 & 4 & 14 & \\
\hline N1 & 1 & 6 & 1 & 11 & \\
\hline $\mathrm{N} 2$ & 0 & 10 & 1 & 7 & \\
\hline N3 & 0 & 1 & 0 & 3 & \\
\hline Type & & & & & 0.588 \\
\hline SCC & 6 & 33 & 6 & 32 & \\
\hline$A C$ & 0 & 1 & 0 & 1 & \\
\hline ASC & 0 & 1 & 0 & 2 & \\
\hline Location & & & & & 0.712 \\
\hline Upper third of esophagus & 1 & 6 & 2 & 5 & \\
\hline Middle third of esophagus & 4 & 21 & 2 & 24 & \\
\hline Distal third of esophagus & 1 & 8 & 2 & 6 & \\
\hline TRG & & & & & 1.000 \\
\hline TRG 1 & 3 & 0 & 1 & 0 & \\
\hline TRG 2 & 3 & 0 & 5 & 0 & \\
\hline TRG 3 & 0 & 3 & 0 & 2 & \\
\hline TRG 4 & 0 & 5 & 0 & 9 & \\
\hline TRG 5 & 0 & 27 & 0 & 24 & \\
\hline \multicolumn{6}{|l|}{ Tumor size } \\
\hline Max size $(\mathrm{cm})$ & $0.428 \pm 0.210$ & $0.222 \pm 0.239$ & $0.178 \pm 0.194$ & $0.132 \pm 0.143$ & 0.376 \\
\hline
\end{tabular}

SCC, squamous cell carcinoma; AC, adenocarcinoma; ASC, adenosquamous carcinoma

\subsection{Development, validation, and performance of a predictive nomogram}

A nomogram was generated from the multimodal logistic regression model derived from DCE-MRI, clinical characteristics and radscore. DCE-MRI and radscore were identified as independent factors of the combined model that the VIF of DCE-MRI and radscore is 5.619, 2.835, 7.905 (Fig. 4). The VIF of the formulate of nomogram as follow:

$$
\boldsymbol{Y}=1 /\left(1+\boldsymbol{e}^{-(\mathbf{2 . 0 6 7 + 1 . 8 0 5} \times \boldsymbol{\beta} 1+-8.843 \times \beta 2)}\right)
$$

NOTE: $\beta 1=$ Rad-score, $\beta 2=$ preVemean.

The results showed that the combined radiomics and DCE-MRI nomogram showed the best discrimination ability (AUC $0.84 ; 95 \% \mathrm{Cl} 0.57-1.00$ in the training set, AUC $0.86 ; 95 \% \mathrm{Cl} 0.74-0.97$ in the test set) that the value of cutoff is 0.5 . The radiomics nomogram alone (Rad-score) was able to discriminate responders from non-responders (AUC $0.82 ; 95 \% \mathrm{Cl}$ $0.59-1.00$ in the training set, AUC $0.79 ; 95 \% \mathrm{Cl} 0.63-0.95$ in the test set), and DCE-MRI nomogram was able to discriminate responders from non-responders (AUC $0.82 ; 95 \% \mathrm{Cl} 0.61-1.00$ in the training set, AUC $0.68 ; 95 \% \mathrm{Cl} 0.36-0.99$ in the test set) (Fig. 5A). The AUC of combined model was the highest among the AUCs of Rad-score and DCE-MRI models, and the differences between combined model and Rad-score or DCE-MRI were not significant ( $P=0.7195$ vs 0.7158 ). The C-index 

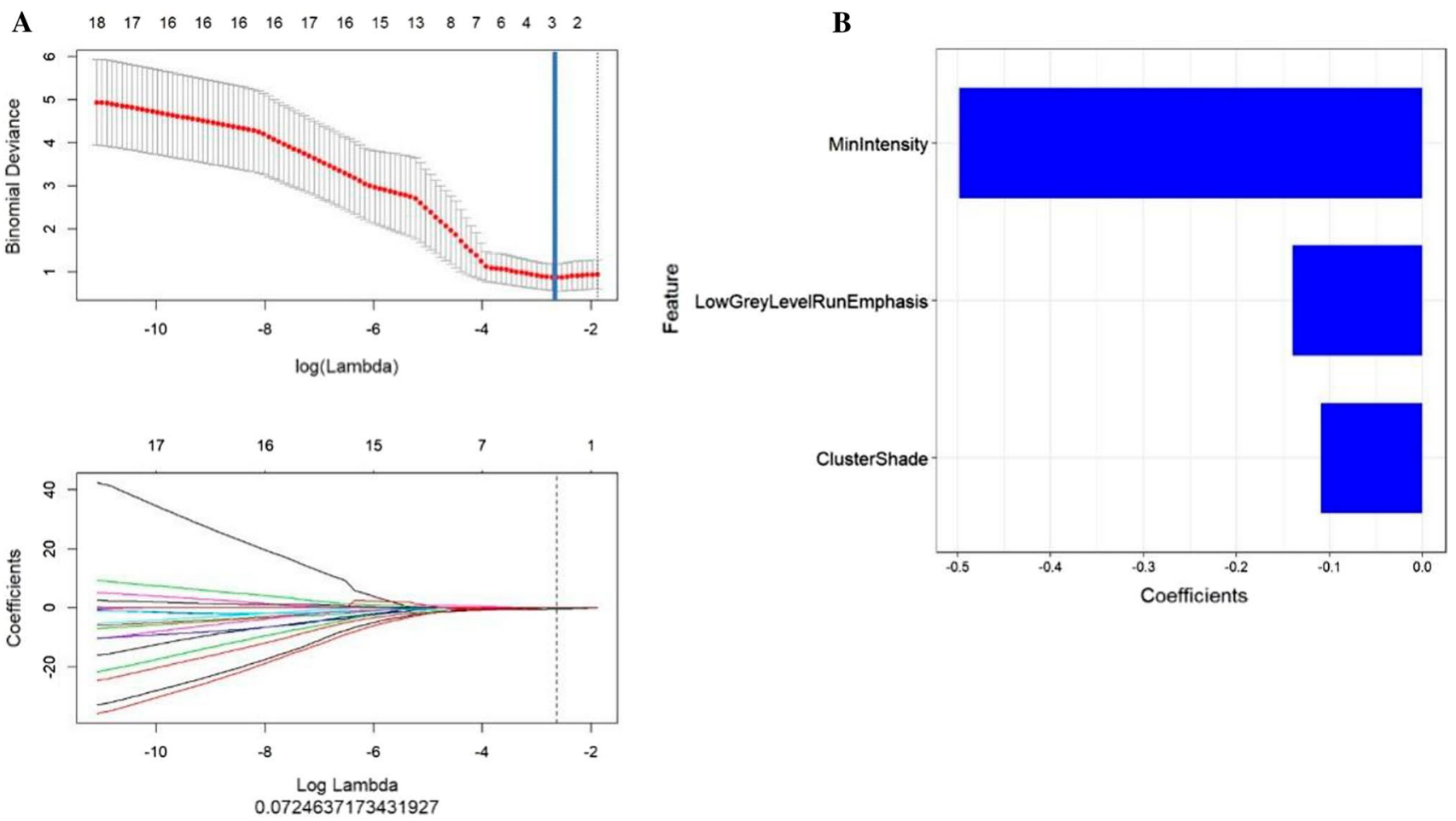

Fig. 2 A selection of response-associated radiomics features via LASSO algorithm, and showing the cross-validation curve. Blue vertical lines were drawn at the optimal value by using tenfold cross-validation and the 1 standard error of the minimum criteria (the 1-SE criteria). An optimal lambda value of 1.181 , with $\log ($ lambda $)=0.0724$, was selected, and 3 nonzero coefficients were chosen. $\mathbf{B}$ the most predictive subset of radiomics features for predicting response to $\mathrm{nCT}$

of nomogram in training group was 0.782 and 0.755 in test group. The Hosmer-Lemeshow test in the combined model showed no significant difference in the goodness-of-fit for the Training set and Testing set $(P=0.729)$.

\subsection{Clinical usefulness of the radiomics nomogram}

As shown in Fig. 5, the clinical impact of the combined DCE-MRI and radiomics nomogram to predict response was observed with maximum utility occurring at 0.95 , and DCA could not be analyzed when combining clinical characteristics. For the majority of risk thresholds, the combined DCE-MRI and radiomics model showed the highest net benefit compared with DCE-MRI and radiomics models (Fig. 5). The combine model that combined radiomics and DCE-MRI showed the highest positive and positive predictive value in both training set $(0.942,0.833$, respectively) and test set $(0.771,1.000$, respectively) (Table 2$)$.

\section{Discussion}

A combined radiomics and DCE-MRI nomogram was developed and validated for the pre-nCT prediction of response in patients with EC, which performed well in discriminating responders from non-responders with an AUC of 0.84 in the training set, and similar discrimination with internal validation ( $A U C=0.86)$. The discrimination abilities in the training and test set were comparable, which implied that the nomogram was reliable in quantifying an individual's risk in non-responders. The combine of radiomics showed the highest PPV in test set (0.971), and the model that combined radiomics and DCE-MRI showed the highest NPV in test set (1.000), however, the specificity of the combined model for test set is the lowest (42.9\%). To our knowledge, this is the first MRI radiomics manuscript for predicting response in EC.

The result demonstrated that radiomics nomogram has the potential to decode intratumor heterogeneity on a macroscopic scale noninvasively and quantitatively $[17,18]$, and genetic alterations or instability can lead to different response to $\mathrm{nCT}$ in EC patients [19]. Further radiogenomic analysis is required to validate this hypothesis. 

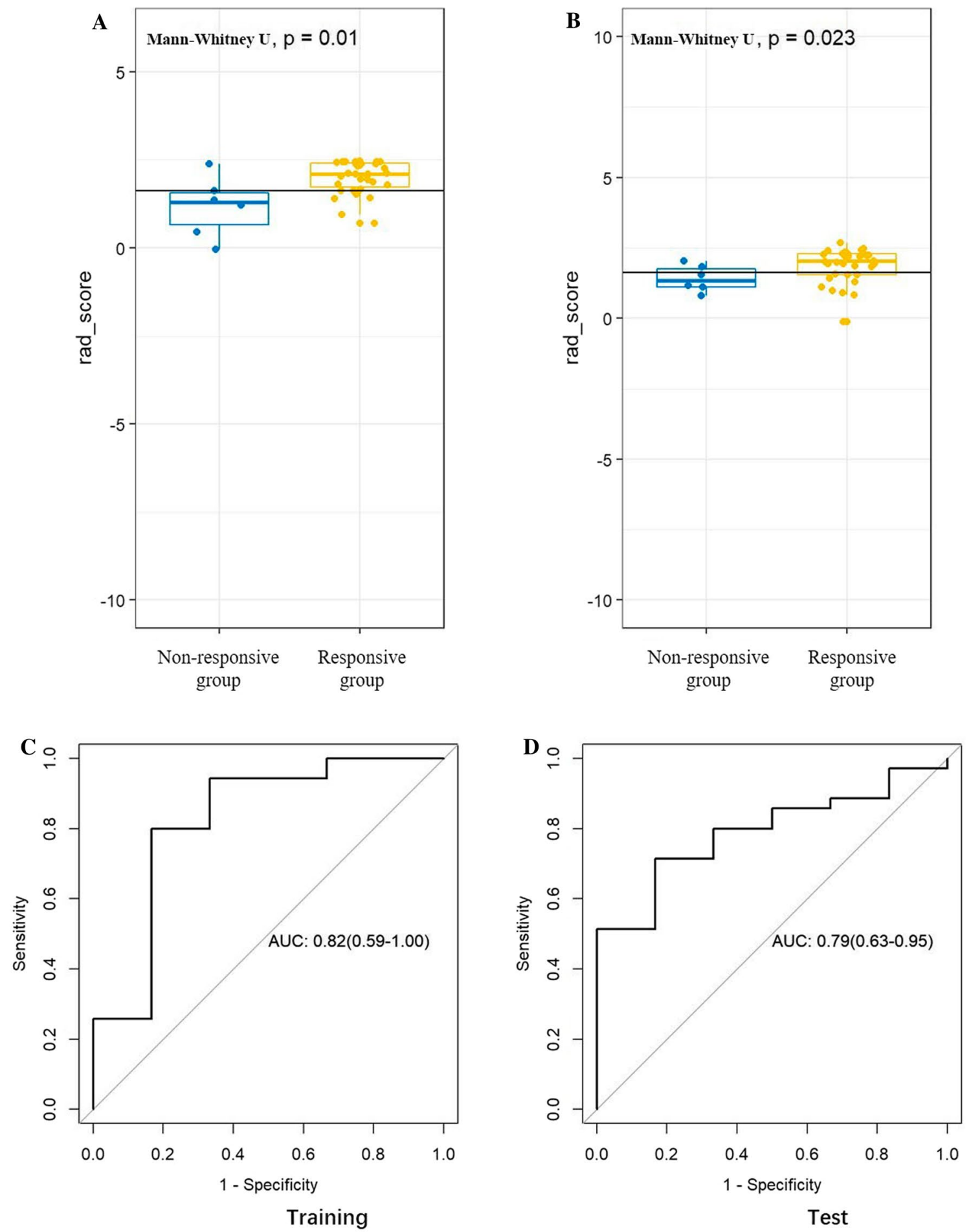

Fig. 3 The radiomics score between responsive group and non-responsive group for each patient in the training set (A) and test set (B). ROCs of radiomics model for predicting response to $\mathrm{nCT}$ on training set $(\mathbf{C})$ and test set (D) respectively

This study showed that the combination of three most significant radiomics features, including MinIntensity, LowGreyLevelRunEmphasis, ClusterShade on pre-nCT MRI, significantly correlated with response and were able to predict response to $\mathrm{NCT}$ in $\mathrm{EC}$ patients. The minimum intensity value is a basic metric that is commonly used to determine the degree of tumor heterogeneity. Cluster analysis or clustering is the task of grouping a set of objects in which objects in the same group (cluster) are more similar (in some sense or another) to each other than to those in other groups (clusters), 
Fig. 4 Developed radiomics nomogram generated by combining the features acquired from LASSO and DCE-MRI. The distribution of predictors and the total points are superimposed on the nomogram scales. The density plots show the distribution of continuous variables, such as radiomics signature and total points. The patient and treatment-related characteristics were included in the radiomics nomogram (Sex: 0 , female; 1, male; P:TRG 1-5; T: T staging)
Points

$\begin{array}{lllllllllll}0 & 10 & 20 & 30 & 40 & 50 & 60 & 70 & 80 & 90 & 100\end{array}$

$\mathrm{V}_{\mathrm{e}}$

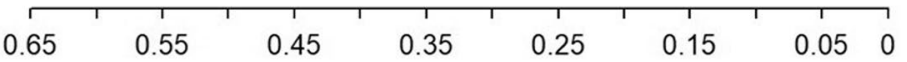

rad_score

$\begin{array}{lllllllllll}-0.2 & 0.2 & 0.6 & & 1 & 1.2 & 1.6 & 2 & 2.2 & 2.6\end{array}$

Total Points

\begin{tabular}{llllllllll}
\hline 0 & 20 & 40 & 60 & 80 & 100 & 120 & 140 & 160 & 180
\end{tabular}

Risk

$\begin{array}{lll}0.1 & 0.4 & 0.9\end{array}$

$\mathbf{A}$
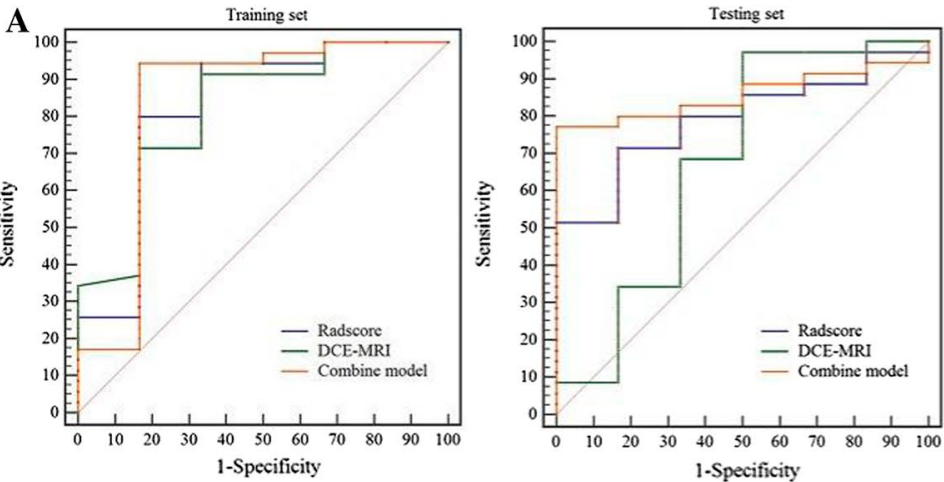

B

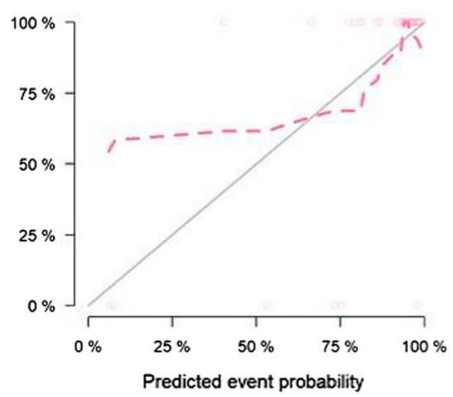

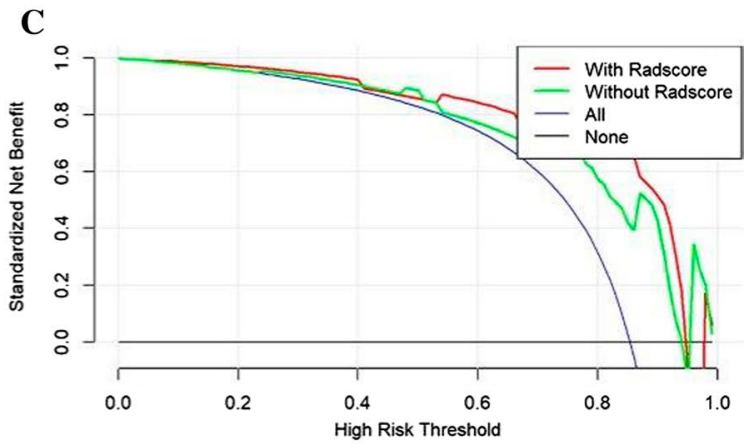

Cost:Benefit Ratio

Fig. 5 A ROC analysis to discriminate responders from non-responders for radscore, clinical model and nomogram. ROC curves for nomogram had the highest area under the ROC curves in both the training set and the test set. B calibration curve of nomogram in the training set and the test set. $\mathbf{C}$ net benefit curves for nomogram compared with models of DCE-MRI andradscore. $Y$ axis means clinical benefit, $X$ axis means the risk of prediction response. None means that none of clinical decision had been taken. All means that random project had been taken. The clinical benefit of nomogram was the highest among nomogram, radscore and clinical model when the clinical risk lower than 0.92

which also correlated with the degree of tumor heterogeneity. The grey level run-length matrix (RLM) is defined as the numbers of runs with pixels of gray level $i$ and run length $\mathrm{j}$ for a given direction $\theta$, which was correlated with the degree of tumor malignant proliferation. The above three radiomics features proved that tumor heterogeneity and angiogenesis were correlated with response to $\mathrm{nCT}$ in EC patients.

Zhang et al. reported that change in the CT value after $\mathrm{nCT}$ can predict therapeutic efficacy in EC patients [20]. T2-weighted imaging and Diffusion-weighted imaging were used to assess the residual tumor after $\mathrm{nCRT}$ for $\mathrm{EC}$ with high sensitivity and low specificity [21, 22]. Intravoxel Incoherent Motion MRI proved useful in early assessment of 
Table 2 Predictive ability of different models

\begin{tabular}{|c|c|c|c|c|c|c|}
\hline & \multicolumn{2}{|l|}{ Radscore } & \multicolumn{2}{|l|}{ DCE-MRI } & \multicolumn{2}{|c|}{ Combine model } \\
\hline & Training set & Testing group & Training set & Testing group & Training set & Testing group \\
\hline AUC & 0.824 & 0.790 & 0.817 & 0.676 & 0.838 & 0.857 \\
\hline Accuracy & 0.805 & 0.732 & 0.878 & 0.854 & 0.927 & 0.805 \\
\hline Youden & 0.633 & 0.548 & 0.581 & 0.471 & 0.776 & 0.771 \\
\hline \multicolumn{7}{|l|}{$95 \% \mathrm{Cl}$} \\
\hline Lower & 0.673 & 0.635 & 0.665 & 0.512 & 0.690 & 0.712 \\
\hline Upper & 0.925 & 0.902 & 0.920 & 0.814 & 0.934 & 0.947 \\
\hline Sensitivity & 0.833 & 0.833 & 0.941 & 0.871 & 0.971 & 1.000 \\
\hline Specificity & 0.800 & 0.714 & 0.571 & 0.500 & 0.714 & 0.429 \\
\hline PPV & 0.417 & 0.333 & 0.914 & 0.971 & 0.942 & 0.771 \\
\hline NPV & 0.966 & 0.962 & 0.667 & 0.962 & 0.833 & 1.000 \\
\hline
\end{tabular}

the response of esophageal squamous cell carcinoma to chemoradiotherapy [23]. PDG-PET response to induction chemotherapy could be a useful imaging biomarker to identify patients with esophageal adenocarcinoma who could benefit from subsequent esophagectomy after chemoradiotherapy [24]. Radiomics had been analyzed for predicting response to neoadjuvant therapy in rectal cancer and cervical cancer patients $[9,10]$. However, to date, little is known about the role of DCE-MRI radiomics in assessing response in patients with EC.

In our study, DCE-MRI was performed by a prototype Radial volumetric interpolated breath-hold examination (VIBE) with the time resolution of $2.4 \mathrm{~s}$ for most phases, which is a new DCE and provides more phases than conventional sequences. Theoretically, Radial VIBE could acquire more information and with higher time resolution than conventional DCE. Heethuis et al. reported that changes in tumor area-under-the-concentration time curve throughout treatment were promising for predicting histopathologic response to $\mathrm{nCRT}$ for EC [4]. Lei et al. reported that $\mathrm{K}_{\text {trans }}$ prior to chemoradiotherapy, and $\mathrm{K}_{\text {trans }}$ and Kep at 3 weeks post-treatment are sensitive prediction parameters that are generated using conventional DCE-MRI [25]. Interesting, in the current study, $17 \mathrm{MRI}$-based texture features on pre-nCT MRI show significance in predicting response of $\mathrm{nCT}$ EC.

The present study also has several limitations. Firstly, the data of both training and validation were acquired from the single institution, and further multicenter validation would be our next work. Secondly, the sample size is small, especially for TRG 1 and 2 (35 non-responders/6 responders), which may lead to a certain degree of bias in the results. Because all patients received only 2 cycles of standard $\mathrm{nCT}$ protocol followed by surgical resection, which may lead to the majority cases to be non-responders, and this $\mathrm{nCT}$ protocol may not have been adequate for $\mathrm{pCR}$. In this study, response rate was $14.6 \%$, which is similar to the report of pathological (20.5\%) response rate [26]. However, no significant difference was seen in 3- and 5-year progression-free survival or 3- and 5-year overall survival. The addition of radiotherapy to neoadjuvant chemotherapy results in higher $\mathrm{RO}$ resection rate and $\mathrm{pCR}$ rate, without significantly impacting survival [27]. Finally, tumor segmentation especially for small lesion could be challenging. However, 2 readers carefully performed tumor segmentation and discrepancy was resolved by consensus.

\section{Conclusions}

To conclude, we constructed a combined radiomics and DCE-MRI nomogram which is able to reliably discriminate tumor response to $\mathrm{nCT}$, and the radiomics features are useful for $\mathrm{nCT}$ response prediction in EC patients. It may provide a convenient tool for clinicians to estimate individuals' risk of non-response to $\mathrm{nCT}$ and to guide treatment personalization for those patients.

Acknowledgements Not applicable.

Authors' contributions JQ: conceptualization, data curation, writing- original draft preparation, conceptualization, methodology, writingreviewing and editing. LM: conceptualization, data curation, writing- original draft preparation, methodology, software, validation. YL: data 
curation. ZW: data curation. JG: data curation. HZ: data curation. XY data curation. HL: conceptualization, data curation. IRK: data analysis/interpretation, writing- original draft preparation, writing- reviewing and editing. JQ: guarantors of integrity of entire study, conceptualization, data curation, writing- original draft preparation, methodology, writing- reviewing and editing. All authors read and approved the final manuscript.

Funding This study has received funding by the Projects of the General Programs of the National Natural Science Foundation of China (No.81972802), Natural Science Foundation of Henan Province (No.182300410355), Henan Province Medical Science and Technology Research Program Provincial Department to jointly build key projects (No.SBGJ202002021), Special funding of the Henan Health Science and Technology Innovation Talent Project (No.YXKC2020011), Henan Province focuses on research and development and promotion (No.212102310133).

Data availability All data generated and analyzed during this study are included in this published article (and its supplementary information files).

\section{Declarations}

Ethics approval and consent to participate The protocol was approved by Henan Cancer Hospital Review Board and informed consent was performed for all the study subjects. All the authors have followed the applicable ethical standards to maintain the research integrity without any duplication, fraud or plagiarism issues.

\section{Consent for publication Not applicable.}

Competing interests The authors declare that they have no competing interests.

Open Access This article is licensed under a Creative Commons Attribution 4.0 International License, which permits use, sharing, adaptation, distribution and reproduction in any medium or format, as long as you give appropriate credit to the original author(s) and the source, provide a link to the Creative Commons licence, and indicate if changes were made. The images or other third party material in this article are included in the article's Creative Commons licence, unless indicated otherwise in a credit line to the material. If material is not included in the article's Creative Commons licence and your intended use is not permitted by statutory regulation or exceeds the permitted use, you will need to obtain permission directly from the copyright holder. To view a copy of this licence, visit http://creativecommons.org/licenses/by/4.0/.

\section{Appendix 1}

See Fig. 6, Table 3 .

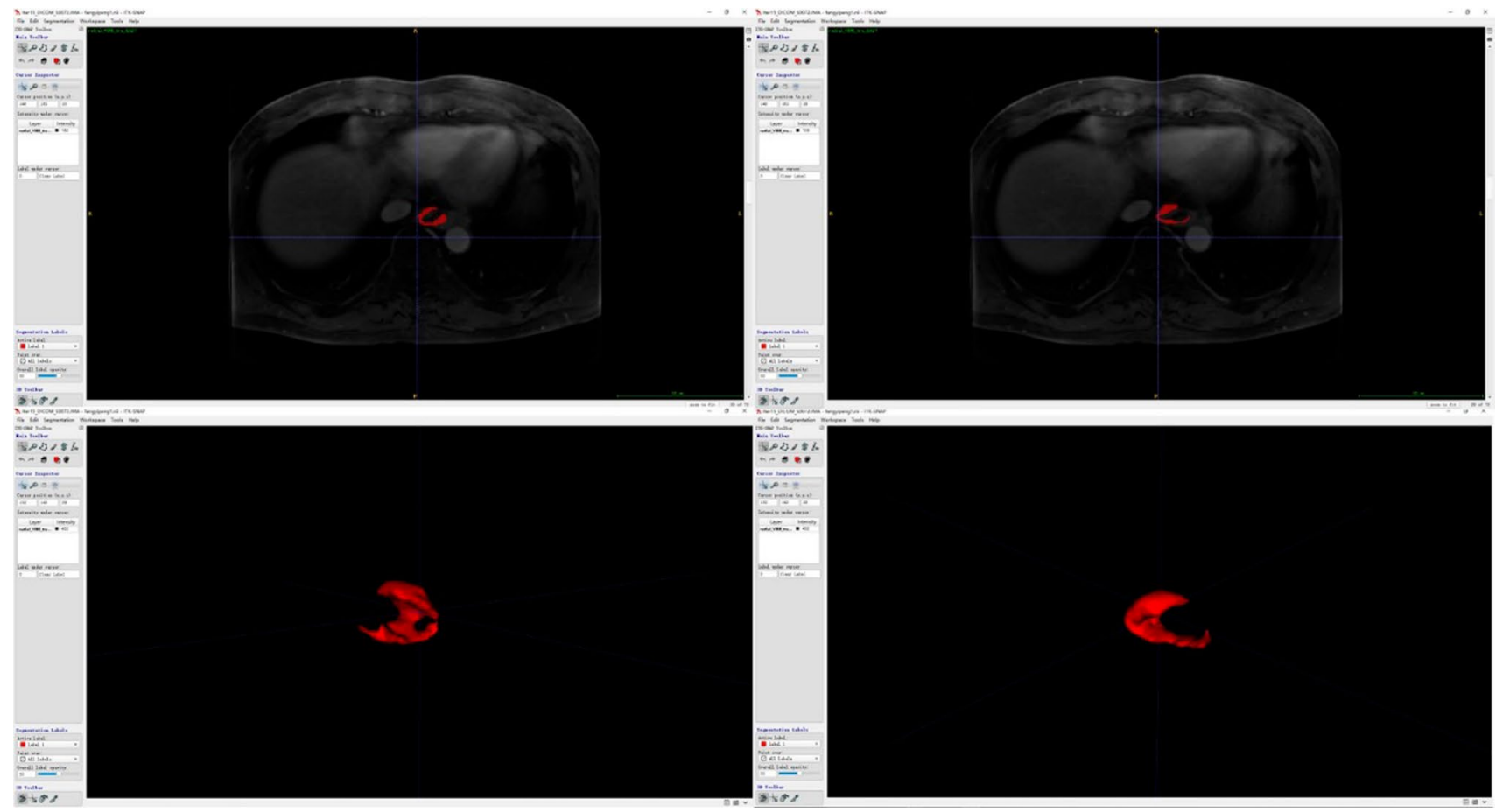

Fig. 6 Tumor Segmentation, and 3D tumors of pre-nCT were segmented 
Table 3 ICC coefficients for all radiomics features

\begin{tabular}{ll}
\hline Radiomics features & Coefficients \\
\hline LongRunEmphasis & 0.999378089664866 \\
GreyLevelNonuniformity & 0.999361834251752 \\
RunLengthNonuniformity & 0.999347482088306 \\
LowGreyLevelRunEmphasis & 0.999280953911486 \\
HighGreyLevelRunEmphasis & 0.999269180347304 \\
ShortRunLowGreyLevelEmphasis & 0.999215212939853 \\
ShortRunHighGreyLevelEmphasis & 0.99919967758075 \\
LongRunLowGreyLevelEmphasis & 0.999161124400232 \\
LongRunHighGreyLevelEmphasis & 0.999137599266105 \\
\hline
\end{tabular}

\section{References}

1. Global Burden of Disease Cancer C, Fitzmaurice C, Allen C, et al. Global, regional, and national cancer incidence, mortality, years of life lost, years lived with disability, and disability-adjusted life-years for 32 cancer groups, 1990 to 2015: a systematic analysis for the global burden of disease study. JAMA Oncol. 2017;3(4):524-48.

2. Allum WH, Blazeby JM, Griffin SM, et al. Guidelines for the management of oesophageal and gastric cancer. Gut. 2011;60(11):1449-72.

3. Boonstra JJ, Kok TC, Wijnhoven BP, et al. Chemotherapy followed by surgery versus surgery alone in patients with resectable oesophageal squamous cell carcinoma: long-term results of a randomized controlled trial. BMC Cancer. 2011;11:181.

4. Heethuis SE, van Rossum PS, Lips IM, et al. Dynamic contrast-enhanced mri for treatment response assessment in patients with oesophageal cancer receiving neoadjuvant chemoradiotherapy. Radiother Oncol. 2016;120(1):128-35.

5. Kuwano H, Nishimura Y, Oyama T, et al. Guidelines for diagnosis and treatment of carcinoma of the esophagus April 2012 edited by the japan esophageal society. Esophagus. 2015;12:1-30.

6. Sjoquist KM, Burmeister BH, Smithers BM, et al. Survival after neoadjuvant chemotherapy or chemoradiotherapy for resectable oesophageal carcinoma: an updated meta-analysis. Lancet Oncol. 2011;12(7):681-92.

7. Xi M, Hallemeier CL, Merrell KW et al. Recurrence risk stratification after preoperative chemoradiation of esophageal adenocarcinoma. Ann Surg. 2017.

8. Vellayappan BA, Soon YY, Ku GY, Leong CN, Lu JJ, Tey JC. Chemoradiotherapy versus chemoradiotherapy plus surgery for esophageal cancer. Cochrane Database Syst Rev. 2017;8:CD010511.

9. Lucia F, Visvikis D, Desseroit MC, et al. Prediction of outcome using pretreatment (18)f-fdg pet/ct and mri radiomics in locally advanced cervical cancer treated with chemoradiotherapy. Eur J Nucl Med Mol Imaging. 2018;45(5):768-86.

10. Horvat $\mathrm{N}$, Veeraraghavan $\mathrm{H}$, Khan $\mathrm{M}$, et al. Mr imaging of rectal cancer: radiomics analysis to assess treatment response after neoadjuvant therapy. Radiology. 2018;287(3):833-43.

11. Qu J, Shen C, Qin J, et al. The mr radiomic signature can predict preoperative lymph node metastasis in patients with esophageal cancer. Eur Radiol. 2018;29(2):906-14.

12. Chen BB, Hsu CY, Yu CW, et al. Early perfusion changes within 1 week of systemic treatment measured by dynamic contrast-enhanced mri may predict survival in patients with advanced hepatocellular carcinoma. Eur Radiol. 2017;27(7):3069-79.

13. Edge SB, Compton CC. The American joint committee on cancer: the 7th edition of the ajcc cancer staging manual and the future of tnm. Ann Surg Oncol. 2010;17(6):1471-4.

14. Wakatsuki K, Matsumoto S, Migita K, et al. Usefulness of computed tomography density of a tumor in predicting the response of advanced esophageal cancer to preoperative chemotherapy. Surgery. 2017;162(4):823-35.

15. Mandard AM, Dalibard F, Mandard JC, et al. Pathologic assessment of tumor regression after preoperative chemoradiotherapy of esophageal carcinoma. Clinicopathol Correlations Cancer. 1994;73(11):2680-6.

16. Huang $\mathrm{YQ}$, Liang $\mathrm{CH}, \mathrm{He} \mathrm{L}$, et al. Development and validation of a radiomics nomogram for preoperative prediction of lymph node metastasis in colorectal cancer. J Clin Oncol. 2016;34(18):2157-64.

17. Lambin P, Leijenaar RTH, Deist TM, et al. Radiomics: the bridge between medical imaging and personalized medicine. Nat Rev Clin Oncol. 2017;14(12):749-62.

18. Gillies RJ, Kinahan PE, Hricak H. Radiomics: images are more than pictures, they are data. Radiology. 2016;278(2):563-77.

19. Ohashi S, Miyamoto S, Kikuchi O, GotoT, Amanuma Y, Muto M. Recent advances from basic and clinical studies of esophageal squamous cell carcinoma. Gastroenterology. 2015;149(7):1700-15.

20. Zhang XY, Yan WP, Sun Y, et al. Ct signs can predict treatment response and long-term survival: a study in locally advanced esophageal cancer with preoperative chemotherapy. Ann Surg Oncol. 2015;22(Suppl 3):S1380-1387.

21. Higano S, Yun X, Kumabe T, et al. Malignant astrocytic tumors: clinical importance of apparent diffusion coefficient in prediction of grade and prognosis. Radiology. 2006;241(3):839-46.

22. Vollenbrock SE, Voncken FEM, van Dieren JM, et al. Diagnostic performance of mri for assessment of response to neoadjuvant chemoradiotherapy in oesophageal cancer. Br J Surg. 2019;106(5):596-605.

23. Zheng H, Ren W, Pan X, et al. Role of intravoxel incoherent motion mri in early assessment of the response of esophageal squamous cell carcinoma to chemoradiotherapy: a pilot study. J Magn Reson Imaging. 2018;48(2):349-58.

24. Xi M, Liao Z, Hofstetter WL, Komaki R, Ho L, Lin SH. Fdg-pet response after induction chemotherapy can predict who will benefit from subsequent esophagectomy after chemoradiotherapy for esophageal adenocarcinoma. J Nucl Med. 2017;158:1756. 
25. Lei J, Han Q, Zhu S, et al. Assessment of esophageal carcinoma undergoing concurrent chemoradiotherapy with quantitative dynamic contrastenhanced magnetic resonance imaging. Oncol Lett. 2015;10(6):3607-12.

26. Zheng Y, Li Y, Liu X, et al. Neoadjuvant chemotherapy followed by minimally invasive esophagectomy versus primary surgery for management of esophageal carcinoma: a retrospective study. J Cancer. 2019;10(5):1097-102.

27. Jing SW, Qin JJ, Liu Q, et al. Comparison of neoadjuvant chemoradiotherapy and neoadjuvant chemotherapy for esophageal cancer: a meta-analysis. Future Oncol. 2019;15(20):2413-22.

Publisher's Note Springer Nature remains neutral with regard to jurisdictional claims in published maps and institutional affiliations. 